\title{
Bioinspired strategy for FWI using frequency alternate grouping
}

\author{
Sérgio Luiz E. F. da Silva (Universidade Federal do Rio Grande do Norte - UFRN), João M. de Araújo (UFRN), Liacir dos \\ Santos Lucena (UFRN) and Gilberto Corso*(UFRN)
}

Copyright 2019, SBGf - Sociedade Brasileira de Geofísica.

This paper was prepared for presentation at the $16^{\text {th }}$ International Congress of the Brazilian Geophysical Society, held in Rio de Janeiro, Brazil, 19-22 August, 2019.

Contents of this paper were reviewed by the Technical Committee of the $16^{\text {th }}$ International Congress of the Brazilian Geophysical Society and do not necessarily represent any position of the SBGf, its officers or members. Electronic reproduction or storage of any part of this paper for commercial purposes without the written consent of the Brazilian Geophysical Society is prohibited.

\section{Abstract}

Full-waveform inversion (FWI) is a challenging methodology in seismic imaging that is strongly evolved in the last years. In the classic approach, all the seismic data simultaneously make contributions equally in the inversion process. However, data recorded at long offsets may contain errors in traveltime estimates if the initial model does not have some information a priori. In this work, we use a bioinspired method to reduce acquisition and data processing using alternate high-pass and low-pass sensors instead of all equal sensors. We test this strategy, in the frequency domain, starting from two initial models: the first is a smoothed version of the true model and the other is a model with little information about the true model. Finally, we show how this approach improve the reconstructed velocity model, principally, when there is little prior information.

\section{Introduction}

Full-waveform inversion (FWI) is a seismic imaging technique that reconstructs the subsurface properties using the information of the propagated mechanical wave reflected by the geologic layers, Tarantola (1984). The FWI strategy consists in solving the wave equation and match the result of the estimated wave with the observed data measured from the geophones Virieux and Operto (2009). A big challenge of the $\mathrm{FWI}$ is that it intrinsically is an illposed inverse problem. In the language of the inversion problems, several models can fit the observed data, and we have to choose the best one.

Usually, FWI uses information from all receivers simultaneously. In this approach, all the seismic traces provide the same contribution to the inversion process. This isn't good when the receiver is too far from the source (large offsets) because information near of the source should contain less uncertainty than information recorded far distant of the source. Several strategies have been adopted to consider seismic traces in the inversion process. Wang (2016) (pp.193-197) proposed an inversion strategy based on grouping traces for tomography for seismic impedance, where the FWI is performed by alternating groups with less information and, at each iteration, increasing the amount of seismic traces to be used in the inversion process.

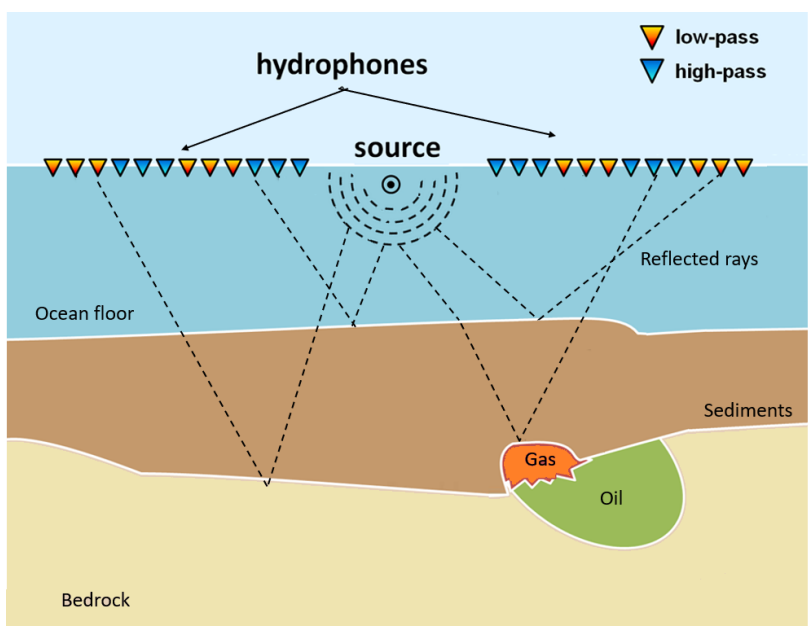

Figure 1: Sketch of the Alternate Frequency Processing (AFP). The acquisition design consists of alternate sets of low-pass and high-pass sensors (represented by triangles of different colors). We note that this strategy can be extended to seismic processing instead of acquisition.

Recently, inspired by bioengineering, it was proposed a new FWI acquisition and processing methodology, the Alternate Frequency Processing (AFP) method de Freitas Silva et al. (2019). The lateral line is an animal organ sense which is mostly present in fish but it is also found in mollusks and amphibians. The lateral line system is basically composed of an array of integrated mechanical sensors Bleckmann and Zelick (2009) which looks like an array of hydrophones. The fish lateral line is basically formed by an arrays of pressure gradient sensors along the fish body which are interconnected by nervous fibers. The fish lateral line has numerous behavioral uses: fish schooling Partridge and Pitcher (1980), sexual matching Satou et al. (1994), orientation to water flows Montgomery et al. (1997) and imaging Hassan (1989). In addition, physiological studies have shown that the mechanical sensors along the lateral line may respond to different frequencies, actual organisms produce different kinds of pass-band sensors that respond better around $10 \mathrm{~Hz}, 40 \mathrm{~Hz}$, or $100 \mathrm{~Hz}$. Weeg and Bass (2002). The work of de Freitas Silva et al. (2019) have explored this idea in the context of frequency domain and it found that the AFP method using a fish inspired sensing method can satisfactorily reconstruct a seismic image, the reconstructed image is as good or even better than the conventional acquisition.

In the traditional seismic acquisition, all sensors are equal in the sense that they respond to the same frequency band. In the AFP method, distinct sets of high frequency and 
low-frequency bands are employed. In biologic systems, the distinct set of sensors are employed, but we call attention that in the seismic imaging context can be more useful to use the alternate high/low pass sensors in the processing methodology. The main advantage of the AFP method is a significant reduction in data acquisition or processing, instead of using a set of $n$ identical sensors, the methodology employs $\frac{n}{2}$ high-pass and $\frac{n}{2}$ low-pass sensors. In this work, we further explore the AFP method distinct threshold frequencies. That means, the frequency limit that defines the sets of low-pass and high-pass sensors.

\section{Methodology}

\section{Basics of Full Wave Inversion}

The Full-Waveform Inversion (FWI) Virieux and Operto (2009) is a technique used to perform high-resolution imaging of the subsurface geological structures. The forward problem of $\mathrm{FWI}$ consists of imaging the propagation of the seismic wave using the model parameters, which in our case are the subsurface wave velocities. In addition, the inverse problem consists of finding the model parameters by fitting the modeled parameters with the observed seismic data.

We employ the acoustic wave equation to describe the wave propagation. For the simplest case of a homogeneous and isotropic medium with velocity $v(x)$, the wave equation is given by:

$$
\left(\nabla^{2}-\frac{1}{v^{2}(x)} \frac{\partial^{2}}{\partial t^{2}}\right) u(x, t)=-s(x, t)
$$

where $x$ is the position, $\nabla^{2}$ the Laplacian operator, $u$ the pressure field, $t$ the time, and the source term is denoted by $s(x, t)$. We remark that equation (1) is a time domain representation of the wave equation. In addition, the physical parameter model consists of $v(x)$, the spatial distribution of wave velocities in the subsurface.

In the paper Tarantola (1984) it was introduced the FWI for the time domain representation while the analogous methodology was extended to the Fourier frequency domain by Pratt et al. (1998). In this study, we utilize the frequency domain because in this representation we can naturally adapt the fish lateral line concepts to FWI language. To create the frequency domain, we apply the Fourier time transformation to equation (1). The resulting frequency-domain acoustic wave equation is written by:

$$
\left(\nabla^{2}+\frac{\omega^{2}}{v^{2}(x)}\right) \hat{u}(x, \omega)=-\hat{s}(x, \omega)
$$

where $\hat{u}$ is the pressure field in the frequency domain, $\omega$ the angular frequency, and $\hat{s}$ the Fourier transform of the source term. The operator $\left(\nabla^{2}+\frac{\omega^{2}}{v^{2}(x)}\right)$ is the impedance matrix Marfurt (1984); Devaney (2012), or the Helmholtz Operator, which depends on the frequency and the medium velocity.

The equation (2) reduces the original wave equation to a system of linear equations which can be written as:

$$
\mathbf{A}(x, z, \omega) \hat{\mathbf{u}}(x, z, \omega)=-\hat{\mathbf{s}}(x, z, \omega)
$$

where $\mathbf{A}$ is the impedance matrix.

\section{The objective function}

The FWI technique is formulated as a non-linear optimization problem, which uses the observed $\mathbf{d}_{o b s}$ seismic data to find the model parameters $(\mathbf{m})$ : the velocity field $v(x)$ Fichtner (2010). The model $(\mathbf{m})$ represents the physical parameters of the subsurface. The solution of the (2) is computed for the full model domain of the wavefield, to compute the misfit we have to estimate the waveforms at the receiver positions for each source. Here, the seismic data is in the frequency domain and takes into account, simultaneously, phase and amplitude. The relationship between the modeled seismic data $\mathbf{d}_{\text {mod }}$ and the model parameters $\mathbf{m}$ is nonlinear, and generically is denoted by an operator $\mathrm{G}$, defined as:

$$
\mathbf{d}_{\text {mod }}=G(\mathbf{m})
$$

The optimization process is conventionally implemented by minimizing the norm of the difference between the observed seismic data $\left(\mathbf{d}_{o b s}\right)$ and the modeled seismic data of the forward problem $\left(\mathbf{d}_{\text {mod }}(\mathbf{m})\right)$ for each source-receiver pair. This difference is our objective function which we intend to minimize.The mathematical representation of this function is done by:

$$
\phi(m)=\sum_{s} \sum_{r} \frac{1}{2}\left\|d_{\mathrm{obs}}-G(\mathbf{m})\right\|_{2}^{2}
$$

where $s$ and $r$ represent the sources and receivers, respectively. In the frequency representation of the inverse problem, equation (5) should be calculated independently for each frequency.

To calculate the minimum of the misfit function, we start from a synthetic physical model, the initial model. The optimization process interactively updates the model parameters by comparing the solution of the forward problem with the observed data. This iterative process starts from the data with the minimum frequency in equation (5), and uses the result as the input model for the next frequency inversion in a sequence from the lowest to the highest frequencies.

\section{The Alternate Frequency Method}

The conventional way to perform acquisition is with a set of identical sensors. Inspired by the fish lateral line system, we propose a different way of calculating the objective function in equation (5), which involves changing the frequency band of operation of the sensors. We called this workflow as the Alternate Frequency Processing (AFP) method.

The AFP method is performed using the mathematical operator $G$ in equation (4) during the sampling procedure. We split the set of sensors into two sets; for frequencies lesser than a threshold frequency, only the first set of receivers is used while for those above the threshold, the other set is employed. In conventional acquisition, all the receivers record all the frequencies (within its operational limits), i. e., the operation in equation (4) is performed with all the receivers and the objective function is evaluated for all the receivers. In the AFP-method, only a part of the data is used for the inversion. For instance, in the case of a $4 \times 4$ AFP method, we use an alternate sequence of 4 high pass 
sensors followed by 4 low pass sensors and again 4 high pass sensors, etc.

The alternate array of sensors is an economical way to deal with the huge amount of information in seismic processing without losing image quality. In this work we perform the inversion with frequencies ranging from $0.5 \mathrm{~Hz}$ to $17 \mathrm{~Hz}$, in the former work de Freitas Silva et al. (2019), for the same frequency range, the threshold frequency was $8 \mathrm{~Hz}$, in the present manuscript we explore threshold frequencies from $3 \mathrm{~Hz}$ to $11 \mathrm{~Hz}$.

\section{Numerical Experiments}

To test the effectiveness of the AFP-method we chose a section of the Overthrust model (Aminzadeh et al. (1997)), see Figure (2).In the numerical experiment we use a classical 9-point finite-differences with absorbing boundary conditions (P. Berenger (1994)) to solve the wave equation. The Helmholtz operator was discretized on a grid with a spacing of $25 \mathrm{~m}$. For all the experiments, it is used a surface acquisition at a depth of $50 \mathrm{~m}$ with 24 equally spaced sources located every $250 \mathrm{~m}$ from $\mathrm{x}=25 \mathrm{~m}$ to 5,775 $\mathrm{m}$. For each source, it was employed 212 receivers located each $50 \mathrm{~m}$ from $\mathrm{x}=0 \mathrm{~m}$ to $6,000 \mathrm{~m}$ at the same depth. The seismic source is a Ricker wavelet centred on $5 \mathrm{~Hz}$.

The inversion process is performed by the multi-scale approach from low to the high frequency in which the output of a low-frequency inversion are subsequently used as the initial model for a higher frequency inversion. This strategy is used to mitigate the non-linearity of the problem (Carey Bunks (1995)). We employed 11 discrete frequencies from $3 \mathrm{~Hz}$ to $13 \mathrm{~Hz}$. We tested three different threshold frequencies $(5,8$ and $11 \mathrm{~Hz})$ in all the experiments. To test the methodology, we implemented eleven AFP-configurations such as $3 \times 3,5 \times 5, \ldots, 19 \times 19$, $21 \times 21$, see tables 1 and 2 . For solving the optimization problem, we used 20 iterations per frequency with the IBFGS method (Nocedal and Wright (2006)).

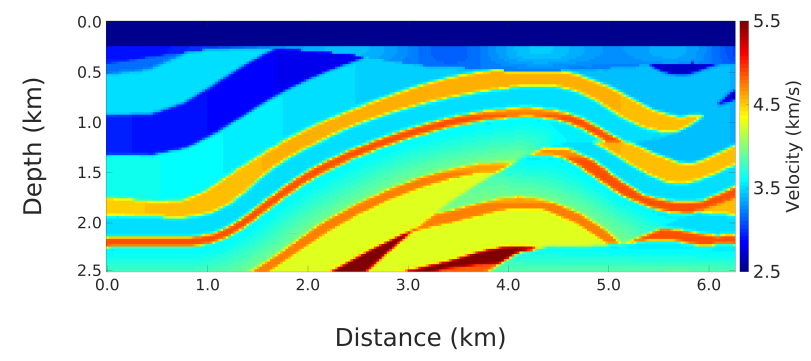

Figure 2: Overthrust velocity model (true model).

The water layer, on top of the model, is assumed to be known and kept constant at $1.5 \mathrm{~km} / \mathrm{s}$. We consider two initial models: the first is a strongly smoothed version of the true model that is obtained from a Gaussian filter of the true model with a standard deviation of $0.5 \mathrm{~km}$, figure $3(\mathrm{a})$. The second initial condition is a linear velocity model which velocity varies from the ocean velocity to the bottom of the model, this model has very little information about the true model, figure $3(b)$. The results of the initial smooth model, figure $3(\mathrm{a})$, are shown in figure 4 . The first three panels refer to AFP$3 \times 3$ configuration using the threshold frequencies: 5,8 and $11 \mathrm{~Hz}$, respectively. The last two panels illustrate

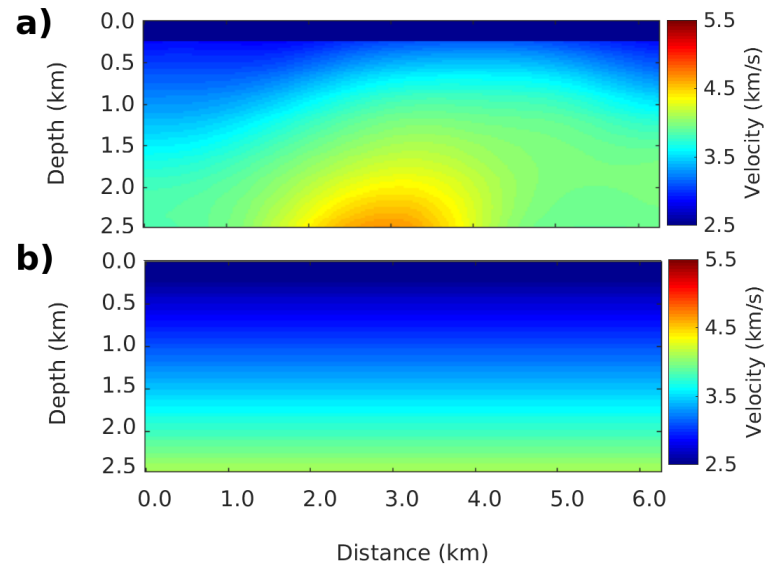

Figure 3: (a) Initial model obtained through a Gaussian filter with a standard deviation of $0.5 \mathrm{~km}$ from the true model. (b) Initial linear velocity model.

the inversion using conventional acquisition and inversion results obtained using information from half-receivers without considering the threshold frequency. In Table 1 , we perform a comparison between the conventional method and the AFP-method quantitatively using the root mean square $(\mathrm{rms})$ and the Pearson correlation coefficient $(r)$ between the reconstructed and true models. Low rms is associated with minor errors. Note that for all experiments, rms values are low and very close to each other and $r$ is very close to 1 . Note that, for all receiver configurations, the estimated models are close to the true model, which means that the reconstructed models are highly-correlated with the true model. In Figure 5 is shown the inversion results starting from the linear velocity model, figure $3(\mathrm{~b})$. The first three figures refer to AFP-15x15 configuration using the threshold frequency of the 5,8 and $11 \mathrm{~Hz}$, respectively. The last two panels illustrate the inversion using conventional acquisition and inversion results obtained using information from halfreceivers without considering the threshold frequency. In comparison, the velocity model estimation obtained using inversion based on the AFP-method is significantly closer to the true model (Figures 5(a), 5(b) and 5(c)).

In both cases, the velocity models are relatively wellreconstructed until $1 \mathrm{~km}$ depth, despite that the AFPmethod generates structures with better definitions than the conventional method. As the initial model has little information on the true model, the inversion process has suffered the effects of cycle-skipping due to uncertainties in the estimation of travel-time of the seismic waves as they crossed the parabolic layer located at approximately $1.0 \mathrm{~km}$ depth. The inversion using the conventional configuration gave greater weight to the errors when using all the information, whereas AFP-method propagates fewer errors at the beginning of the acquisition. This result is reinforced by Table 2 where we have shown a comparison between the conventional method and the AFP-method quantitatively. The rms for the reconstructed model through conventional configuration is much larger than the method proposed in this work. Moreover, all reconstructed models using AFP-method shows a strong correlation with the true model $(0.5981 \leq r \leq 0.7271)$ while the simulation with the 


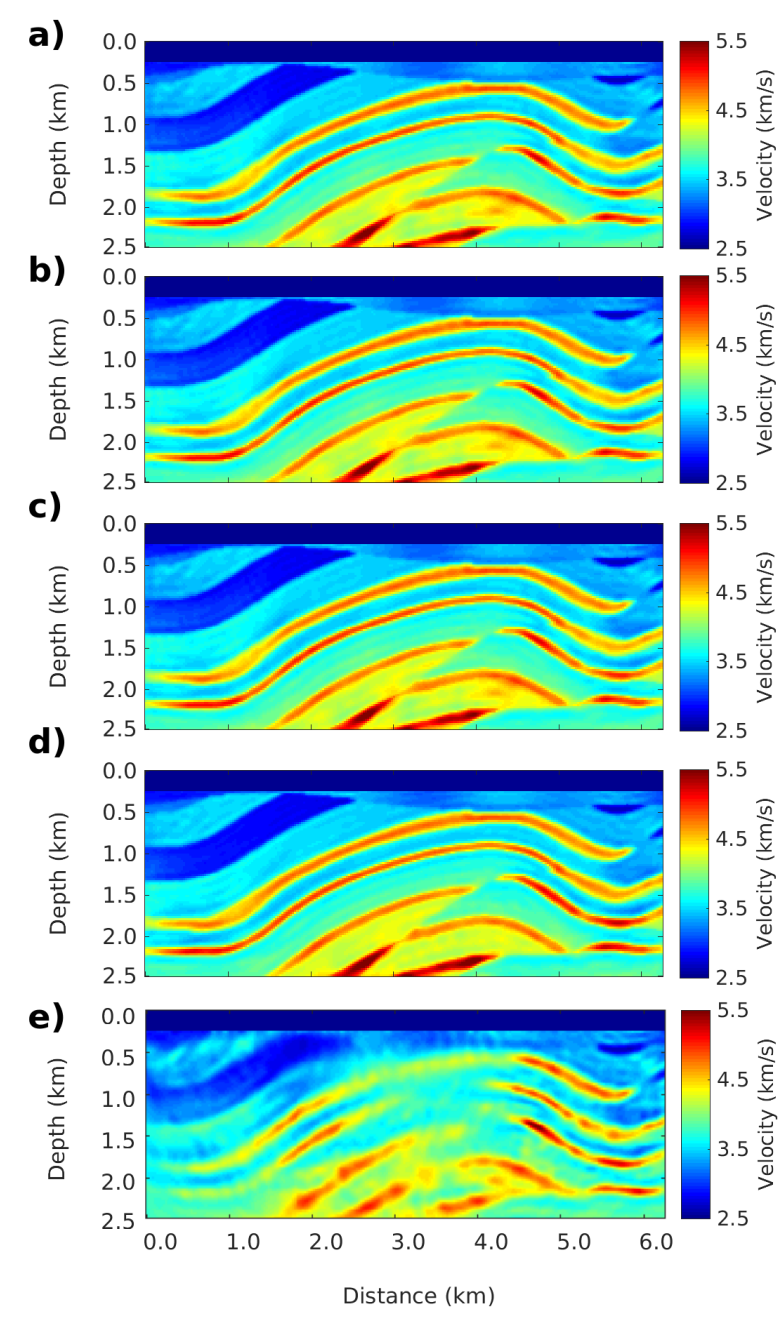

Figure 4: Results from the smooth initial model, figure 3(a). We employ the AFP-3x3 with a threshold frequency: (a) $5 \mathrm{~Hz}$; (b) $8 \mathrm{~Hz}$ and (c) $11 \mathrm{~Hz}$. (d) Conventional. acquisition. (e) Using information from half-receivers without considering the threshold frequency.

conventional method shows a correlation close to zero with the true model $(r=0.0462)$.

Note that, FWI using the AFP-method yields better results starting from a bad initial models and it's as good as the conventional method starting from a good initial model. For each initial model, a further 27 experiments were performed, but all reconstructed models were very similar, for this reason, they aren't shown.

In Figures 6 and 7 we compare the objective function of the conventional method with the AFP-method for two initial models. In all cases shown (and in others not shown), the AFP objective function converges faster or comparable than the conventional inversion. For low frequencies, both methods have similar convergences, because at low frequencies, the problem is less non-linear than in high frequencies. Moreover, for high frequencies, the AFP method shows a better than the conventional method disregarding the initial model. So, our approach reduces

\begin{tabular}{cccc}
\hline array & $t f$ & $r m s$ & $r$ \\
\hline conventional & - & $\mathbf{0 . 1 7 7 4}$ & $\mathbf{0 . 9 6 3 6}$ \\
& 5 & 0.2004 & 0.9533 \\
$3 \times 3$ & 8 & 0.1998 & 0.9535 \\
& 11 & 0.1998 & 0.9535 \\
$5 \times 5$ & 5 & 0.1991 & 0.9538 \\
& 8 & 0.1983 & 0.9542 \\
$7 \times 7$ & 11 & 0.1984 & 0.9542 \\
& 5 & 0.2017 & 0.9526 \\
& 8 & 0.2017 & 0.9526 \\
$9 \times 9$ & 11 & 0.2004 & 0.9533 \\
& 5 & 0.2025 & 0.9522 \\
$11 \times 11$ & 8 & 0.2035 & 0.9517 \\
& 11 & 0.2044 & 0.9513 \\
& 5 & 0.2052 & 0.9510 \\
$13 \times 13$ & 8 & 0.2060 & 0.9505 \\
& 11 & 0.2040 & 0.9515 \\
& 5 & 0.2026 & 0.9522 \\
& 8 & 0.2016 & 0.9526 \\
$15 \times 15$ & 11 & 0.2000 & 0.9534 \\
& 5 & 0.2028 & 0.9521 \\
$17 \times 17$ & 8 & 0.2050 & 0.9510 \\
& 11 & 0.2047 & 0.9512 \\
& 5 & 0.2072 & 0.9499 \\
& 8 & 0.2041 & 0.9514 \\
& 11 & 0.2020 & 0.9524 \\
& 5 & 0.2027 & 0.9523 \\
& 8 & 0.2042 & 0.9514 \\
& 11 & 0.2042 & 0.9514 \\
& 5 & 0.2030 & 0.9520 \\
& 8 & 0.2071 & 0.9500 \\
& 11 & 0.2086 & 0.9492 \\
\hline & & & \\
& &
\end{tabular}

Table 1: Table with the main statistics of the numerical experiments using as initial model the smooth model, figure 3(a). $t f$ is the threshold frequency, rms is the Root Mean Square and $r$ is a measure of similarities between models.

significantly the computational cost and the processing time requiring less iterations and less memory than the standard inversion.

\section{Conclusions}

In this work, we explore an innovative method of seismic imaging inspired in fish lateral line bioengineering developed in de Freitas Silva et al. (2019). Instead of the trivial all equal sensor line used in exploratory geophysics the cited work develops an array of alternate sensors with different frequencies. This concept is worked inside the FWI method which, in the frequency domain representation, show an ideal framework to implement the methodology Pratt et al. (1998).

The sensing strategy of using regions of low/high frequency band frequency sensors is a consequence of hundreds of millions of years of evolution of animal life. We remark that fishes are among the oldest vertebrate animal taxon, which dates back to 450 million years ago Nelson (2006). In this sense, we can hypothesize that nature had plenty of time to adapt an optimal acquisition mechanism for the fish lateral line. Moreover, we understand the sensing acquisition mechanism in a neuroscience perspective, as a mechanism with the ability to acquire, compute, and 


\begin{tabular}{cccc} 
array & $t f$ & $r m s$ & $r$ \\
\hline conventional & - & 8.2207 & 0.0462 \\
$3 \times 3$ & 5 & 0.5507 & 0.7050 \\
& 8 & 0.5507 & 0.6995 \\
$5 \times 5$ & 11 & 0.5518 & 0.6981 \\
& 5 & 0.5300 & 0.7184 \\
& 8 & 0.5324 & 0.7130 \\
$7 \times 7$ & 11 & 0.5330 & 0.7123 \\
& 5 & 0.5357 & 0.7111 \\
& 8 & 0.5370 & 0.7080 \\
$9 \times 9$ & 11 & 0.5351 & 0.7110 \\
& 5 & 0.5446 & 0.7077 \\
& 8 & 0.5418 & 0.7066 \\
$11 \times 11$ & 11 & 0.5430 & 0.7059 \\
& 5 & 0.5547 & 0.7014 \\
& 8 & 0.5450 & 0.7077 \\
$13 \times 13$ & 11 & 0.5426 & 0.7118 \\
& 5 & 0.5473 & 0.7078 \\
& 8 & 0.5369 & 0.7132 \\
& 11 & 0.5348 & 0.7153 \\
$15 \times 15$ & 5 & 0.5386 & 0.7098 \\
& 8 & 0.5464 & 0.7044 \\
$17 \times 17$ & 11 & 0.5490 & 0.7022 \\
& 5 & 0.5301 & 0.7181 \\
& 8 & 0.5235 & 0.7232 \\
& 11 & 0.5205 & 0.7271 \\
& 5 & 0.5427 & 0.7075 \\
& 8 & 0.5448 & 0.7080 \\
& 11 & 0.5475 & 0.7045 \\
& 5 & 0.5490 & 0.7038 \\
& 8 & 0.5509 & 0.7038 \\
& 11 & 0.5529 & 0.7019 \\
\hline & & &
\end{tabular}

Table 2: Table with the main statistics of the numerical experiments using as initial model the linear velocity model, figure 3(b). tf is the threshold frequency, $r m s$ is the Root Mean Square and $r$ is a measure of similarities between models.

process information. The animal nervous system has evolved to work as a complex system that has to sense the environment, process the information, and respond to the stimulus in a fast and efficient way Dethier and Stellar (1964). In this sense, the alternate frequency acquisition should be an evolved strategy to maximize velocity processing with a minimum of sensory information.

\section{Acknowledgements}

The authors acknowledge the support of Conselho Nacional de Desenvolvimento Científico e Tecnológico, CNPq - Brazil. The authors also gratefully acknowledge the support from Shell Brasil through the "New Methods for Full Waveform Inversion" project at Universidade Federal do Rio Grande do Norte and the strategic importance of the support given by ANP through the R\&D levy regulation.

\section{References}

Aminzadeh, F., Jean, B., and Kunz, T., 1997, 3-d salt and overthrust models: Society of Exploration Geophysicists.

Bleckmann, H., and Zelick, R., 2009, Lateral line system of fish: Integrative zoology, 4, no. 1, 13-25.

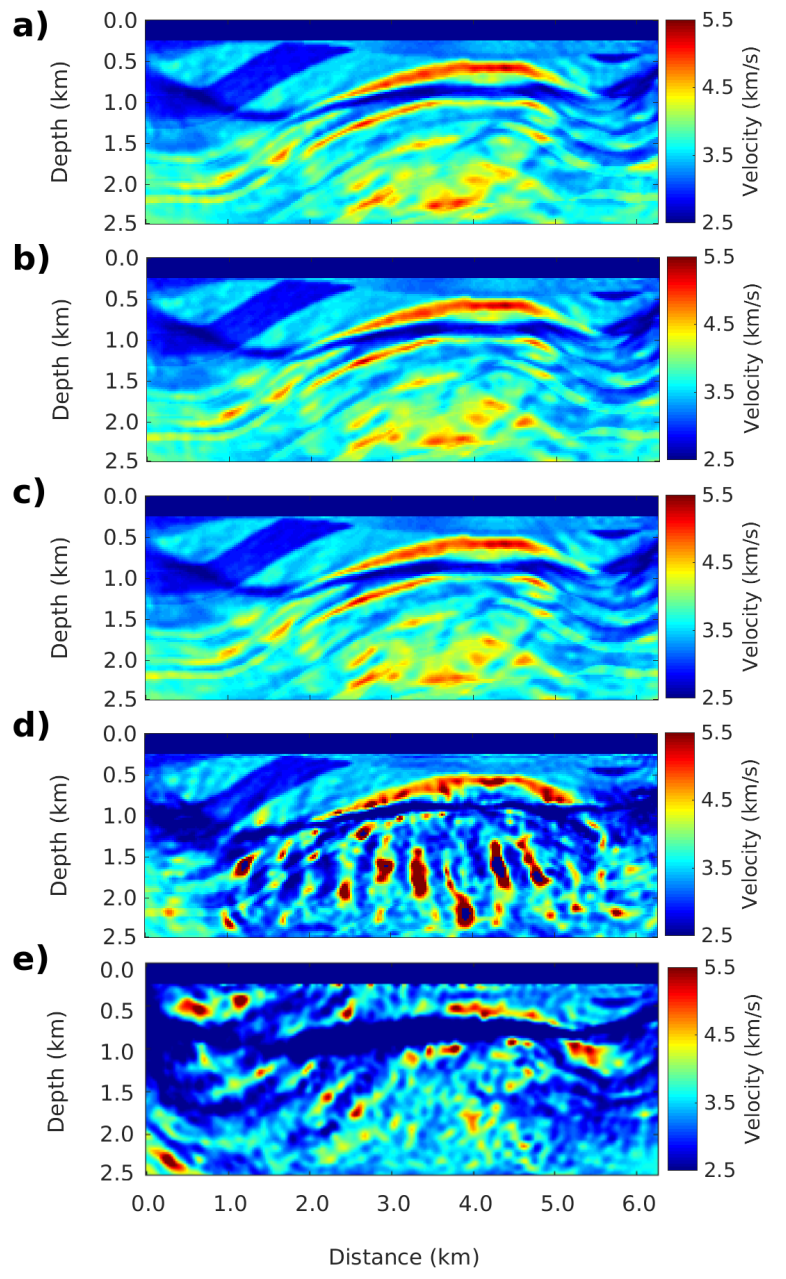

Figure 5: Results from the linear velocity initial model, figure $3(b)$. We show the AFP-15x15 arrange with a threshold frequency: (a) $5 \mathrm{~Hz}$; (b) $8 \mathrm{~Hz}$ and (c) $11 \mathrm{~Hz}$. (d) Conventional acquisition. (e) Using information from halfreceivers without considering the threshold frequency.

Carey BunksFatimetou M. Saleck, S. Z. G. C., 1995, Multiscale seismic waveform inversion: Geophysics, 60, no. $5,1457-1473$.

de Freitas Silva, F. W., da Silva, S. L. E. F., Henriques, M. V. C., and Corso, G., 2019, Using fish lateral line sensing to improve seismic acquisition and processing:.

Dethier, V. G., and Stellar, E., 1964, Animal behaviour - its evolutionary and neurological basis: Prentice Hall, Inc.

Devaney, A., 2012, Mathematical foundations of imaging, tomography and wavefield inversion: Cambridge University Press.

Fichtner, A., 2010, Full Seismic Waveform Modelling and Inversion: Springer Verlag.

Hassan, 1989, Hydrodynamic imaging of the surroundings by the lateral line of the blind cave fish anoptichthys jordani: Neurobiology and Evolution (ed. S. Coombs, P. Gorner and H. Munz) New York: Springer-Verlag. 


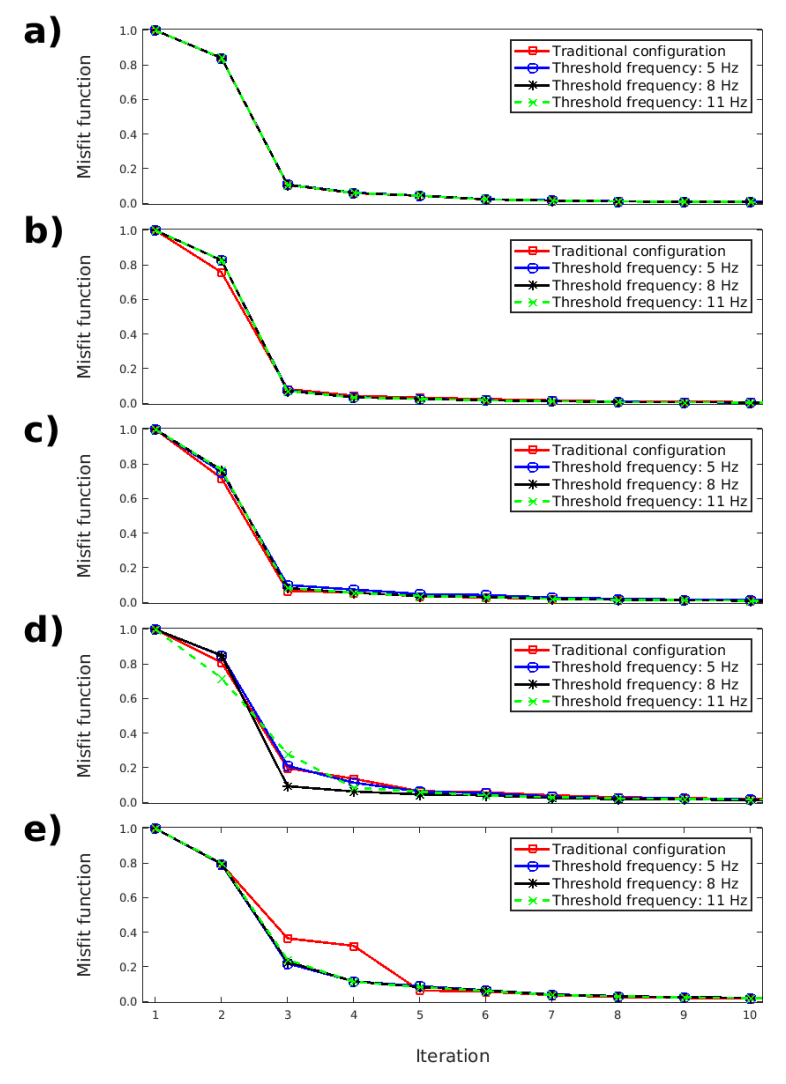

Figure 6: Comparison between different objective functions for the inversion process from the smooth initial model using AFP-5X5. Inversion frequency (a) $3 \mathrm{~Hz}$; (b) $5 \mathrm{~Hz}$; (c) $8 \mathrm{~Hz}$; (d) $11 \mathrm{~Hz}$ and (e) $13 \mathrm{~Hz}$.

Marfurt, K. J., 1984, Accuracy of finite-difference and finite-element modeling of the scalar and elastic wave equations: Geophysics, 49, no. 5, 533.

Montgomery, J. C., Baker, C. F., and Carton, A. G., 1997, The lateral line can mediate rheotaxis in fish: Nature, 389, 960-963.

Nelson, J. S., 2006, Fishes of the world - fourth edition: John Wiley and Sons, Inc.

Nocedal, J., and Wright, S. J., 2006, Numerical optimization: Springer, New York, NY, USA, second edition.

P. Berenger, J., 1994, A perfect matched layer for the absorption of electromagnetic waves: Journal of Computational Physics, 114, 185-200.

Partridge, B. L., and Pitcher, T. J., 1980, The sensory basis of fish schools: relative roles of lateral line and vision: Journal of comparative physiology, 135, no. 4, 315-325.

Pratt, G., Shin, C., and Hicks, 1998, Gauss-newton and full newton methods in frequency-space seismic waveform inversion: Geophysical Journal International, 133, no. 2, 341-362.

Satou, M., Takeuchi, H.-A., Nishii, J., Tanabe, M., Kitamura, S., Okumoto, N., and Iwata, M., 1994, Behavioral and a)

b)

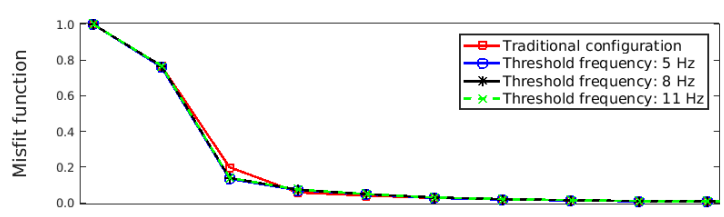

c)
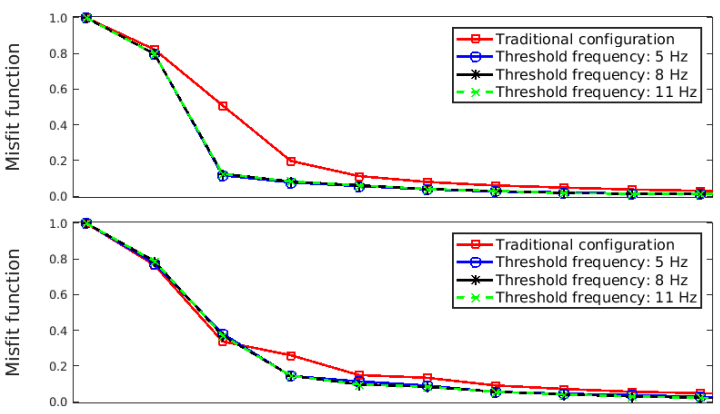

d)

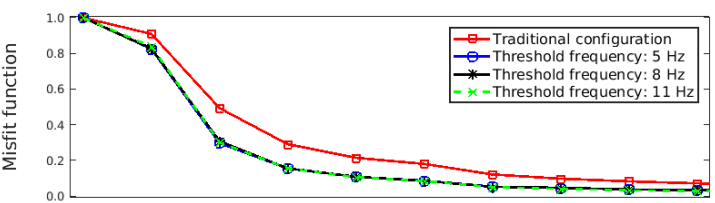

e)

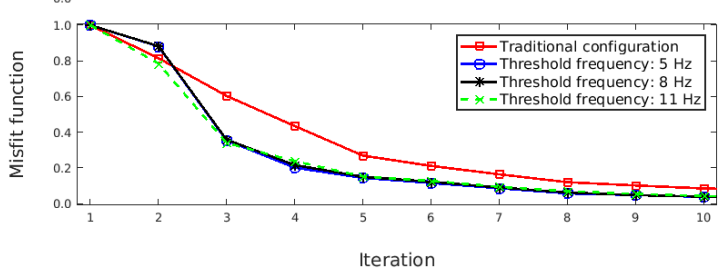

Figure 7: Comparison between different objective functions for the inversion process from the linear velocity model using AFP-5X5. Inversion frequency (a) $3 \mathrm{~Hz}$; (b) $5 \mathrm{~Hz}$; (c) $8 \mathrm{~Hz}$; (d) $11 \mathrm{~Hz}$ and (e) $13 \mathrm{~Hz}$.

electrophysiological evidences that the lateral line is involved in the inter-sexual vibrational communication of the himé salmon (landlocked red salmon, oncorhynchus nerka): J. Comparative Physiology, 174, 539-549.

Tarantola, A., 1984, Inversion of seismic reflection data in the acoustic approximation: Geophysics, 49, no. 8, 1259-1266.

Virieux, J., and Operto, S., 2009, An overview of full-waveform inversion in exploration geophysics: Geophysics, 74, WCC1-WCC26.

Wang, Y., 2016, Seismic inversion: Theory and applications: Wiley-Blackwell.

Weeg, M. W., and Bass, A. H., 2002, Frequency response properties of lateral line superficial neuromasts in a vocal fish, with evidence for acoustic sensitivity: Journal of Neurophysiology, 88, 1252. 